\title{
The surgical treatment of thoracic metastases in ovarian cancer
}

\author{
Alice SAVESCU ${ }^{1}$, Irina BALESCU ${ }^{2}$, Nicolae BACALBASA ${ }^{3}$ \\ ${ }^{1}$ Department of Obstetrics and Gynecology, University Emergency Hospital, Bucharest, Romania \\ 2 „Ponderas" Hospital, Bucharest, Romania \\ ${ }^{3}$ "Carol Davila“ University of Medicine and Pharmacy, Bucharest, Romania
}

\begin{abstract}
Ovarian cancer still represents one of the most aggressive malignancies affecting women worldwide. Unfortunately many patients are still diagnosed in an advanced stage of the disease when distal metastases are already present. When it comes to thoracic involvement in advanced stage ovarian cancer, this may develop via peritoneal, hematogenous and lymphatic route. This is a literature review of the most commonly seen involvements in ovarian cancer and of the most appropriate surgical procedures which might be performed in order to increase the rate of complete cytoreduction.
\end{abstract}

Keywords: advanced stage ovarian cancer, peritoneal seeding, metastasectomy,

diaphragmatic resection, supradiaphragmatic lymphadenectomy

\section{INTRODUCTION}

Ovarian cancer is one of the most lethal diseases, representing $2 \%$ of all malignancies and $4 \%$ of malignancies in women. (1) Majority of cases are diagnosed in advanced stages of the disease, up to $50 \%$ of patients being diagnosed in FIGO stage III and $13 \%$ in stage IV. In patients diagnosed with metastatic ovarian cancer the 5 year survival rate varies between $28-33 \%$. (2)

Advanced ovarian cancer usually presents with peritoneal seeding, distant metastasis, mainly hepatic and pulmonary, multiple adenopathy, neoplastic pleuritis.

\section{Peritonectomy and diaphragmatic resections}

Many patients have diaphragmatic involvement. In these cases, in order to increase the rate of complete cytoreduction and secondarily to improve the overall survival, various surgical procedures have been imagined. Nowadays there are three types of surgical procedures managing diaphragmatic disease: the first one is a superficial destruction or ablation (SD) which consists of coagulation of very small and tiny nodules $(\leq 2-3 \mathrm{~mm})$, the second procedure is diaphragmatic peritonectomy (stripping, DS) defined as dissection and resection of the peritoneum presenting tumoral seeding while the third type is the full thickness diaphragm resection (FTDR) which means diaphragm muscle resection or central tendon resection including the overlying peritoneum and pleura. After pleurectomy and before performing a full-thickness suture a catheter is placed in the pleural cavity. The last procedure in associated with pneumothorax and a higher rate of pleural effusion. (3) In most cases diaphragmatic peritonec- 
tomy is associated with full thickness diaphragm resection. (4) Depending on the local diaphragmatic extension of the metastases the adequate type of surgery is selected. (1)

As presented before advanced ovarian cancer is usually associated with residual tumor and diaphragm metastasis (5) and an important percent of these patients are submitted to cytoreductive surgery due to the fact that residual disease remains the most important independent prognostic factor. (6)

In some cases the metastases are located not only in the diaphragam but also in the liver. New modern techniques such as robotic resection of liver and full-thickness diaphragm are mentioned. (7)

Traditionally, optimal cytoreduction of ovarian cancer has been limited to abdominal disease. While there is an increase in morbidity and mortality with upper abdominal surgery, the outcomes appear to justify the risks. (6) While liver and diaphragmatic resection are becoming more common, lung disease pose unique challenges.

\section{Pulmonary resections for ovarian cancer metastases}

There are few studies referring to pulmonary metastasectomy in ovarian cancer. A substantial number of studies refer to gynecologic cancerthis review takes in consideration only those where ovarian cancer is included. (8)

Although in time the indication and the adequate type of resection during pulmonary metastasectomy from different types of cancers has been debated some questions still remain. (9) This is also the case of pulmonary mestastasis from ovarian cancer. (8) In order to help the practitioner guidelines have been published for the management of pulmonary metastasectomy from different cancers. Specifically guidelines for pulmonary metastsectomy from ovarian cancer haven't been published yet, but there are studies conducted on this subject which are similar with those presented for breast and colonic cancers. Generally, pulmonary metastasectomy originating from gynecologic malignancies should be taken in consideration in patients (1) with no evidence of recurrent disease at their primary, (2) with isolated pulmonary metastases, (3) in patients with adequate pulmonary reserve, and (4) in the absence of uncontrollable extrathoracic metastatic disease. (8)

Pulmonary metastasectomy is a controversial subject in the oncology field. (10) For some histophatological subtypes such as epithelial cancer significant survival benefit after the resection of metastases was proven, but clear inclusion criteria in this group could not be specified. (11)

The most common type of pulmonary metastasectomy in different cancers includes anatomical pulmonary resections, wedge resections and laser excisions. (12) The procedure of choice for treatment of pulmonary metastatic disease is pulmonary wedge excision. Extensive pulmonary resection, such as pneumonectomy, should be discouraged in these patients with poor prognostic factors. (8) Segmentectomy for pulmonary metastases is a new technique that seems promising. It is less invasive than lobectomy, negative margins can be achieved to ensure complete resection of lesions and an adequate pulmonary function is easier to be preserved, but large studies regarding segmentectomy in ovarian cancer pulmonary metastases haven't been published yet. (12)

Even in cases submitted to complete primary cytoreduction, recurrent ovarian tumors might develop and a secondary cytoreduction might be needed. Koichi et al reported the case of a patient diagnosed with liver and diaphragmatic metastases originating from an ovarian clear cell tumor which were successfully resected; after a year she developed pulmonary metastases and right lower lobectomy was performed. (8)

Few cases of direct extension of the diaphragm metastasis into the lung are presented; in these cases en bloc full-thickness diaphragmatic resection including a portion of lung tissue is considered optimal solution. (13)

In rare cases after several debulking procedure metastases are found in the liver with direct extension into diaphragm and lung. In these cases the tumor is resected en bloc with atypical hepatectomy full thickness diaphragm resection and lung resection. A right lower lobe wedge resection of the tumor mass en bloc is mentioned. (14)

Right pulmonary metastasectomy is more frequently performed than left one because usually pulmonary metastases are found in the right lung, but are some case reports that mention metastases from ovarian granulosa cell tumor in the left lung in which a partial resection of the left lung was performed. (15)

Distant metastases in multiple organs can be found in ovarian cancer. This is the case of a patient with immature teratoma of the ovary that developed lung, liver and brain metastases. The 
management of the case was sequential resections of all three metastatic areas which led to a ten year survival (16).

Thoracic metastases of ovarian cancer are common, the thorax being the third site of dissemination after liver and diaphragm. Although noninvasive techniques as positron emission tomography with 18F-fluorodeoxyglucose - (18F) FDG-PET - have been developed to investigate this area, sometimes is difficult to establish the exact pathology of pleural or lung nodules. Thoracoscopy is a technique performed when the suspicion of metastasis exists and when nodules on visceral pleura are found are resected. Metastases from ovarian granulosa cell tumor have been diagnosed in visceral pleura by this method. (17)

\section{Supradiaphragmatic lymph node involvement in ovarian cancer}

At this moment the enlargement of supradiaphragmatic lymph nodes is not always associated with malignancy and even if they are positive the prognostic significance and staging indicator isn't known. (18) Because of these findings several techniques to explore the cardiophrenic space have been developed. A new method to biopsy these lymph nodes is transabdominal cardiophrenic lymph node dissection (CPLND). (19) It is considered more useful than video-assisted thoracic surgery (VATS) in advanced ovarian cancers with cardiophrenic involvement in which biopsy can be performed at the same time with cytoreductive surgery.

Usually cardiophrenic lymph nodes are associated with advanced, extensive intrathoracic disease represented by right-sided pulmonary and pleural dissemination. (20) Isolated adenopathy of the mediastinum is rare while isolated bilateral adenopathies have been never described before in association with ovarian cancer. A few studies noted the findings of isolated abnormal cardiophrenic lymph nodes in different types of ovarian cancer. $(18,19)$ Two cases of isolated adenopathy without lung or pleural involvement from papillary ovarian cancer are presented which were removed completely through the help of video-assisted thoracic surgery. $(18,19)$ Mediastinal cyst with benign aspect without pleuro-pulmonary pathological findings on CT evaluation was identified at a woman with personal history of ovarian cystadenocarcinoma which was identified after en-block resection with free margins as mediastinal recurrence of the ovarian cancer. (20)
In general when lymph nodes are identified with metastases from ovarian cancer the patient is already diagnosed with the primary disease. In this way even though clinical malignancy characters can't be found on the modified lymph nodes the suspicion of the dissemination is put into question. Isolated metastatic papillary pattern lymph nodes without personal history of cancer were identified on immunohistochemistry after surgical biopsy before the intraabdominal ovarian serous carcinoma manifests. (21)

Mediastinal metastases from high-grade serous ovarian carcinoma are mentioned in different case reports. (22) Video-assisted thoracoscopic surgery performed for an irregularly-shaped calcified chest mass along the right cardiac border (identified as low-grade papillary serous carcinoma from ovary), multiple pulmonary nodules identified on $\mathrm{CT}$ at a woman with pelvic mass measuring $6 \times 7 \mathrm{~cm}$ found an unresectable mediastinal mass because of her involvement in the phrenic nerve and the superior vena cava that compresses the tight atrium. A right middle lobe wedge resection was performed for pulmonary metastases and mediastinal mass was treated with Cyberknife radiosurgery. (23)

Systematic mediastinal lymph dissection is accepted to be performed for complete resection in non-small cell lung cancer. (24) In other types of cancers with lung metastases when pulmonary metastasectomy is performed systemic mediastinal lymphadenectomy is not a practice. When modified mediastinal lymph are found in preoperative (thoracic CT, PET) investigations and when the suspicion of malignancy appears a series of procedure including mediastinoscopy are performed although positive results are rare. At this moment the prognostic significance of positive lymph nodes with pulmonary metastasectomy has not been established. (25) It appears that the presence of lymph node metastases influences the survival and tumor recurrence. Due to these factors some authors sustain the benefits of systematic lymph node dissection at the time of pulmonary metastasectomy for metastatic ovarian carcinoma but still a standard therapeutic protocol has not been established. (26)

Mediastinal involvement in patients with ovarian cancer is rare (27) and is associated with advanced disease stage and poor prognosis. Prevascular mediastinal lymph node biopsy showed metastatic ovarian adenocarcinoma from serous papillary ovarian adenocarcinoma. (28) 
Malignant pleural effusion from metastatic ovarian cancer usually necessitates the initiation of chemotherapy. Symptomatic patients may require thoracocentesis. Other invasive methods used for pleural effusion used are tube thoracostomy, small-bore catheters (pigtail catheters), both of them with the advantage of drainage and pleurodesis.

Patients with suspected advanced ovarian cancer and moderate to large pleural effusions may be evaluated through video-assisted thoracic surgery. Video-assisted thoracic surgery has an important role in the management of these cases. Depending on the findings at video-assisted thoracic surgery multiple types of procedure as pleural cytology, chest tube drainage, adheolysis, directed biopsies can pe performed. Pleural nodes greater than $1 \mathrm{~cm}$ are resected as part of intrathoracic cytoreduction with the help of this procedure. $(24,29)$

Thoracic metastasis in ovarian cancer represents an open problem for oncology. Pulmonary metastasectomy can improve survival in selected patients, but little information exists regarding this group of patients. Although general practice is mentioned: patients (1) with no evidence of recurrent disease at the primary site, (2) with pulmonary metastases that are limited in number, (3) with the patient who has adequate pulmonary reserve, and (4) when no evi- dence of extrathoracic metastatic disease exists or can be controlled, information about the cell type of the primary tumor, the exact number and localization of metastases does not exist. Also the surgical technique used for pulmonary resection poses questions in some cases: wedge resections, lobectomy or segmentectomy? Information about regional lymph nodes are even scarcer. Recommendations regarding the indications and potential therapeutic role of mediastinal lymphadenectomy or sampling have not been defined.

Several prognostic indicators have been identified such as short interval between resection of the primary tumor and the first pulmonary metastasesectomy and the finding of metastases in mediastinal lymph nodes.

\section{CONCLUSIONS}

Although a standard therapeutic protocol has not yet been established complete macroscopic resection of thoracic lesions originating from ovarian cancer seems to be the most efficient option in order to improve survival. However more studies are still needed in order to assess the most important prognostic factors and to decide in which cases these resections might provide a more significant improvement of long term outcomes.

\section{$\overline{\text { BIBLIOGRAFIE }}$}

1. Aletti G.D., Dowdy S.C., Podratz K.C., Cliby W.A. Surgical treatment of diaphragm disease correlates with improved survival in optimally debulked advanced stage ovarian cancer. Gynecologic Oncology. 2006; 100(2):283-287.

2. www.cancercenter.com/ovarian-cancer/ statistics/tab/ovarian-cancer-survivalstatistics/

3. Tsolakidis D., Amant F., Van Gorp T., Leunen K., Neven P., Vergote I. Diaphragmatic surgery during primary debulking in 89 patients with stage IIIB-IV epithelial ovarian cancer. Gynecologic Oncology. 2010; 116(3):489-496.

4. www.esgo.org/Education/ Publishinglmages/119.pdf

5. Cliby W., Dowdy S, Feitoza S.S., Gostout B.S., Podratz K.C. Diaphragm resection for ovarian cancer: technique and short-term complications. Gynecologic Oncology. 2004; 94(3):655-660.
6. Pathiraja P.N.J., Garruto-Campanile R., Tozzi R. Diaphragmatic Peritonectomy versus Full Thickness Diaphragmatic Resection and Pleurectomy during Cytoreduction in Patients with Ovarian Cancer. Int J Surg Oncol. 2013; 2013: 876150.

7. Robert W., Brudie L.A., Rakowski J.A., Ahmad S. Robotic-assisted resection of liver and diaphragm recurrent ovarian carcinoma: Description of technique. Gynecologic Oncology. 2011; 120(3):419-422.

8. Koichi Fujiu, Hideaki Miyamoto, Satoshi Hashimoto, Nobuyasu Suzuki, Yoshinao Takano, Yasushi Teranishi, Hideo Sakuma, Hiroyuki Suzuki. A case of diaphragmatic clear cell carcinoma in a patient with a medical history of ovarian endometriosis. International Journal of Clinical Oncology. 2010; 15(5):489-492.
9. Higashiyama M., Tokunaga T., Nakagiri T., Ishida D., Kuno H., Okami J. Pulmonary metastasectomy: outcomes and issues according to the type of surgical resection. Gen Thorac Cardivasc Surg. DOI 10.1007/s11748-015-0544-9

10. Ito H., Nakayama H. Surgical management of pulmonary metastases. Gan To Kagaku Ryoho. 2010 Feb; 37(2):200-3.

11. Kycler W., Kubiak A., Laski P., Cybulski Z., Perz H. Lung surgery for metastatic epithelial tumours. Postepy Hig Med Dosw (Online). 2015 Mar 5;69:277-84.

12. Vodicka J., Spidlen V., Simanek V., Safranek J., Skalicky T., Fichtl J., Mukensnabl P., Rousarova M. Surgical therapy of pulmonary metastases - 10-year results. Bratisl Lek Listy. 2013; 114(4):218-24.

13. dos Santos L.A., Modica I., Flores R.M., D’Angelica M., Aghajanian C., Chi D.S., Nadeem R. Abu-Rustum. En bloc resection 
of diaphragm with lung for recurrent ovarian cancer: A case report. Gynecologic Oncology. 2006; 102(3):596-598.

14. Edwards J.M., Lowery W., Secord A.A. Quinary debulking for epithelial ovarian cancer. Journal of Surgical Case Reports Volume. 2012,7:7-7.

15. Matsui H., Omiya H., Takami K., Mishima H., Ban K., Kodama Y., Kuriyama K., Sekimoto M. Pulmonary metastasis of an ovarian granulosa cell tumor resected 11 years before. Kyobu Geka. 2014 Sep; 67(10):904-7.

16. Kurata A., Hirano K., Nagane M., Fujioka Y. Immature teratoma of the ovary with distant metastases: favorable prognosis and insights into chemotherapeutic retroconversion. Int J Gynecol Pathol. 2010; 29(5):438-44.

17. Liu Y., Ren T., Feng F.Z., Wan X.R., Shen K., Xiang Y. Clinical analysis of 15 cases of malignant ovarian germ cell tumors with lung metastasis. Zhonghua Fu Chan Ke Za Zhi. 2012 Jan; 47(1):40-4.

18. Ragusa M., Vannucci J., Capozzi R., Daddi N., Avenia N., Puma F. Isolated cardiophrenic angle node metastasis from ovarian primary. report of two cases. J Cardiothorac Surg. 2011; 6: 1.

19. Heon Jong Yoo, Myong Cheol Lim, Yong Jung Song, Yuh-Seock Jung, Sun Ho Kim, Chong Woo Yoo, Sang-Yoon Park. Transabdominal cardiophrenic lymph node dissection (CPLND) via incised diaphragm replace conventional video-assisted thoracic surgery for cytoreductive surgery in advanced ovarian cancer. Gynecologic Oncology. 2013;129(2):341-345

20. Lococo F., Charpentier C.H., Guinet C., Brandolini J., Alifani M., Regnard J.F. Mediastinal recurrence from ovarian cystadenocarcinoma presenting as pleuro-pericardial cyst. European Review for Medical and Pharmacological Sciences. 2014; 18: 2094-2096.

21. Zannoni G.F., Vellone V.G., Distefano M.G., Fadda G., Scambia G. Ovarian serous carcinoma presenting with mediastinal lymphadenopathy 20 months before the intraabdominal mass: role of immunohistochemistry. Gynecol Oncol. 2007 Feb; 104(2):497-500.

22. Mayadagli A., Ekici K., Akcicek M., Korkmaz T., Eser M., Kayipmaz S.S. Metastasis to mediastinal lymph nodes from ovarian cancer: A case report. Case Study Case Rep. 2012; 2(4): 129-136.

23. Raidooa S., Singh S., Redline R., DeBernardo $\mathbf{R}$. Cyberknife radiotherapy and anastrozole for the treatment of advanced progressive low-grade papillary serous ovarian carcinoma: A case report. Gynecologic Oncology Case Reports. 2013; 6:42-44.

24. Juretzka M.M., Abu-Rustum N.R., Sonoda Y., Downey R.J., Flores R.M., Park B.J., Hensley M.L., Barakat R.R., Chi D.S. The impact of video-assisted thoracic surgery (VATS) in patients with suspected advanced ovarian malignancies and pleural effusions. Gynecol Oncol. 2007 Mar; 104(3):670-4.
25. Clavero J.M., Deschamps C., Cassivi S.D., Allen M.S., Nichols F. III, Barrette B.A., Larson D.R., Pairolero P.C. Gynecologic Cancers: Factors Affecting Survival After Pulmonary Metastasectomy. 2006; 81(6), 2004-2007.

26. Ercan S., Nichols F.C. III, Trastek F., Deschamps C., Allen M.A., Miller D.L., Schleck C.D., Pairolero P.C. Prognostic significance of lymph node metastasis found during pulmonary metastasectomy for extrapulmonary carcinoma. 2004; 77(5):1786-1791.

27. Zannoni G.F., Vellone V.G., Distefano M.G., Fadda G., Scambia G. Ovarian serous carcinoma presenting with mediastinal lymphadenopathy 20 months before the intraabdominal mass: role of immunohistochemistry. Gynecologic Oncology. 2007; 104(2):497-500.

28. Nwankwo N., Gilman A.D., Mirrakhimov A.E., Iroegbu N.A. Mediastinal involvement in ovarian cancer. BMJ Case Reports 2013; doi:10.1136/bcr-2013-009795.

29. Diaz J.P., Abu-Rustum N.R., Sonoda Y., Downey R.J., Park B.J., Flores R.M., Chang K., Leitao M.M. Jr, Barakat R.R., Chi D.S. Video-assisted thoracic surgery (VATS) evaluation of pleural effusions in patients with newly diagnosed advanced ovarian carcinoma can influence the primary management choice for these patients. Gynecol Oncol. 2010 Mar;116(3):483-8. 